\title{
SOME FOURIER DIVISION PROBLEMS
}

\author{
R. E. EDWARDS \\ (Received 11 November 1968; revised 17 September 1969) \\ Communicated by J. B. Miller
}

\section{Introduction}

Let $T$ denote the circle group, $C$ the set of continuous complex-valued functions on $T$, and $A$ the set of $f \in C$ having absolutely convergent Fourier series:

$$
\sum_{n \in I}|\hat{f}(n)|<\infty,
$$

$I$ standing for the set of integers.

It would seem that G. E. Shilov ([1]; $\left[2_{\mathrm{II}}\right]$, p. 187) was the first to note the existence of functions $f, h \in A$ such that (to speak loosely) $h / f$ belongs to $C$ but not to $A$. Whenever this happens, the set $Z(f)$ of zeros of $f$ is necessarily nonvoid; and then, unless $Z(f)$ is nowhere dense, there is ambiguity involved in the possible continuous extension(s) of $h / f$ (which has domain $T \backslash Z(f)$ ). In order to avoid such ambiguities, we reformulate this type of problem in a manner making no explicit reference to division.

As a starting point, we remark that a well-known consequence of applying the Gelfand theory to $A$ is the following statement (see $\S 4$ for more details).

0.1 If $\Phi$ is a nonvoid subset of $A$ having a (possibly void) set $Z(\Phi)$ of common zeros, and if $g$ is a complex-valued function on $T$ which vanishes on some neighbourhood in $T$ of $Z(\Phi)$ and satisfies $f g \in A$ for every

In particular:

$$
f \in \Phi, \text { then } g \in A \text {. }
$$

0.2 If $f, h \in A$, and if $h$ vanishes on some neighbourhood of $Z(f)$, then the function

belongs to $A$.

$$
g=0 \quad \text { on } \quad Z(f),=h / f \text { on } T \backslash Z(f)
$$

These results suggest the following sort of problem. Given a nonvoid subset $\Phi$ of $A$, under what conditions does there exist $g \in C$ such that $f g \in A$ for every $f \in \Phi, g=0$ on $Z(\Phi)$, and $g \notin A$ ?

We shall in this note discuss and obtain a partial answer to the above problem 
in a more general setting, $T$ being replaced by a more general group and/or the spaces $C$ and $A$ being varied. (Still further generalisation is possible.) We shall, however, obtain a general existential result only in the case in which $\Phi$ is countable. The details of the context in which we shall work are given in $\S 1$, the general theorem in $\S 2$, and applications of this general theorem appear in $\S 3$. A constructive approach applying in a special situation is described in $\S 5$.

The general Theorem 2.1 will by specialisation (the details of which appear in 3.3) yield the following sample result.

0.3 Suppose that $\Phi$ is a countable subset of $A$ such that

either $\Phi$ is contained in the smallest closed ideal in $A$ whose zero-set is $Z(\Phi)$ (which is the case whenever $Z(\Phi)$ is a spectral synthesis set),

or every element of $\Phi$ satisfies a Lipschitz condition of order $\frac{1}{2}$.

Then there exist functions $g$ satisfying a Lipschitz condition of order $\frac{1}{2}$ which vanish on $Z(\Phi)$, which satisfy $f g \in A$ for every $f \in \Phi$, and which even so do not belong to $A$.

This result is best-possible in the sense that any function satisfying a Lipschitz condition of order greater than $\frac{1}{2}$ necessarily belongs to $A$ (see $\left[3_{1}\right]$, p. 167).

ACKNOWLEDGEMENTS. The author would like to express his thanks to a referee for general comments and especially for the present formulation of 3.3, which is an improvement over the original version.

\section{Definitions and assumptions}

1.1 Henceforth $G$ will denote an infinite Hausdorff compact Abelian group and $C(G)$ the complex Banach space of continuous complex-valued functions on $G$, the norm on $C(G)$ being

$$
\|f\|_{\infty}=\sup \{|f(x)|: x \in G\} .
$$

If $P$ and $Q$ denote subsets of $C(G)$, we write $P Q$ or $P \cdot Q$ for the set of product functions $f g$ with $f \in P$ and $g \in Q$.

We shall consider several normed (complex) linear spaces, each of which is assumed to be (algebraically) a linear subspace of $C(G)$, the corresponding injection map being continuous. $F, X, Y$ and $Z$ will be normed linear spaces in this sense, of which $X$ and $Z$ are assumed furthermore to be complete.

$\mathscr{T}$ denotes a topology on $X$ weaker than the norm topology. (In most of the later applications, these two topologies coincide.)

1.2 $Y$ is assumed to be a subset of $X$ and to be such that

$$
\text { the closed unit ball } B \text { in } Y \text { is } \mathscr{T} \text {-closed in } X \text {, }
$$




$$
F \cdot Y \subset Z,
$$

and

$$
\|f g\|_{Z} \leqq c|| f\left\|_{F}\right\| g \|_{Y}
$$

for every $f \in F, g \in Y, c$ being independent of $f$ and $g$.

1.3 Write $X_{U}$ for the set of $f \in X$ such that $\operatorname{supp} f \subset U$, where supp $f$ denotes the support of $f$, and $Y_{U}$ for $X_{U} \cap Y$.

The final assumption concerning $X$ and $Y$ is that, for each nonvoid open subset $U$ of $G$, there exists $g \in X_{U} \backslash Y$ and a sequence $\left(g_{r}\right)_{r=1}^{\infty}$ extracted from $Y_{U}$ such that

$$
\mathscr{T}-\lim _{r \rightarrow \infty} g_{r}=g
$$

and

$$
m=\sup _{r}\left\|g_{r}\right\|_{X}<\infty .
$$

1.4 We shall say that $(\Phi, K)$ is an $F$-admissible pair if $\Phi$ is a nonvoid subset of $F$ and the following two conditions are fulfilled:

(a) $K$ is a nonopen closed subset of $G$ which is contained in the zero-set $Z(\Phi)$ (= set of common zeros of elements) of $\Phi$;

(b) every $f \in \Phi$ is the limit in $F$ of a sequence $\left(f_{n}\right)_{n=1}^{\infty}$ extracted from $F$, each $f_{n}$ vanishing on some (possibly $n$-dependent) neighbourhood in $G$ of $K$.

In the main theorem to follow we shall be concerned with $F$-admissible pairs $(\Phi, K)$ in which $\Phi$ is countable: we shall term these briefly (iff oddly) countable F-admissible pairs.

It is of course trivial that $(\Phi, K)$ is $F$-admissible whenever $\Phi \subset F, K$ is a nonopen closed subset of $G$, and $K$ is contained in the interior of $Z(\Phi)$. In general, $(\Phi, Z(\Phi))$ will not be $F$-admissible; but in all the examples we shall consider later $(\Phi, K)$ will be $F$-admissible provided $K$ satisfies (a) and provided every element of $\Phi$ is 'sufficiently smooth' and/or vanishes 'sufficiently strongly' at each point of $K$ (the precise meaning of the phrases in quotation marks depending upon the normed linear space $F$ ); see 2.5 below.

\subsection{Remarks}

(i) The assumptions made in 1.1 and 1.2 concerning $F, X, Y, Z$ are not minimal; they have been chosen so as to be at once reasonably simple and adequate for the applications in $\S 3$ below.

(ii) In all the applications we make, $F$ and $Y$ are complete. In any such case $\left(1.2^{\prime}\right)$ is a consequence of (1.2) and 1.1 on account of general theorems (see, for 
example, [6], Theorems 6.4.2 and 7.7.9). On the other hand, the direct verification of (1.2) will in most cases involve the proof of $\left(1.2^{\prime}\right)$ as well; cf. 3.6 .2 and 3.7.2 below.

\section{The main theorem}

2.1 THEOREM. The notations and hypotheses being as in 1.1-1.3, suppose that $(\Phi, K)$ is a countable $F$-admissible pair. Denote by $E$ the linear space formed by those functions $g \in X$ such that

$$
g=0 \quad \text { on } K, \quad f g \in Z \quad(f \in \Phi)
$$

make E into a topological linear space having a base at 0 formed of the sets

$$
\left\{g \in E: N_{j}(g) \leqq \varepsilon\right\},
$$

where $j$ runs over the positive integers and $\varepsilon$ over the positive numbers,

$$
N_{j}(g)=\|g\|_{X}+\sum_{i=1}^{j}\left\|f_{i} g\right\|_{z}
$$

for every $g \in E$, and where $i \rightarrow f_{i}$ is a surjection of $\{1,2, \cdots\}$ onto $\Phi$. Then:

(i) $E$ is a Fréchet space containing every element of $Y$ which vanishes on $K$;

(ii) the set $E_{0}=E \backslash Y$ is comeagre and everywhere dense in $E$;

(iii) there exist functions $g \in X$ satisfying (2.1) and $g \notin Y$.

\subsection{REMARKS}

(1) Given 2.1(i), it follows that $E$ is a Baire space and so that any comeagre subset of $E$ is everywhere dense in $E$; see [6], 0.3.16.

(2) Plainly, 2.1(ii) entails that $E_{0}$ is nonvoid, so that any $g \in E_{0}$ satisfies 2.1(iii).

These remarks show that, to prove 2.1 , it suffices to establish 2.1 (i) and the following assertion:

(iv) $E \backslash E_{0}=E \cap Y$ is a meagre subset of $E$.

2.3 Proofs of 2.1(i) AND 2.2(iv)

As to 2.1(i), it is first of all clear from 1.2 that $E$ contains every element of $Y$ which vanishes on $K$, and that $E$ is a metrisable locally convex space; thus it remains only to show that $E$ is complete. But suppose $\left(g_{k}\right)_{k=1}^{\infty}$ to be a Cauchy sequence extracted from $E$. The definition of the topology on $E$ combines with the assumed completeness of $X$ and $Z$ (see 1.1) to show that there exist $g \in X$ and $h_{i} \in Z(i=1,2, \cdots)$ such that

$$
\lim _{k} g_{k}=g \text { in } X \text { and } \lim _{k} f_{i} g_{k}=h_{i} \text { in } Z
$$


for $i=1,2, \cdots$. These limiting relations hold a fortiori in the sense of uniform convergence on $G$ (see 1.1 again) and so entail that $g=0$ on $K$ and that $h_{i}=f_{i} g$ for every $i$. Consequently, $g \in E$ and $\lim _{k} g_{k}=g$ in $E$. This proves completeness of $E$.

The proof of 2.2(iv) proceeds by contradiction, assuming that $E \cap Y$ is nonmeagre in $E$. Indeed, if this be assumed, the relation

$$
E \cap Y=\bigcup_{v=1}^{\infty}(E \cap v B)
$$

which is an immediate consequence of (1.1), coupled with the fact that each $E \cap v B$ is closed in $E$ (since each $v B$ is norm-closed in $X$ ), would show via Baire's theorem ([6], 0.3.16) that some $E \cap v B$ has interior points relative to $E$. Since $E \cap v B$ is convex and balanced, it is accordingly a neighbourhood of zero in $E$. This signifies the existence of integers $v$ and $j$ and a real number $\delta>0$ such that

$$
g \in E, N_{j}(g) \leqq \delta \Rightarrow g \in v B,
$$

or, what is equivalent, such that

$$
E \subset Y \text { and }\|g\|_{Y} \leqq \nu \delta^{-1} N_{j}(g) \quad(g \in E) .
$$

Hence, by (1.2), $\left(1.2^{\prime}\right)$ and (2.4) we have for every $f \in F$ and $g \in E$ :

$$
f g \in Z, \quad\|f g\|_{Z} \leqq c v \delta^{-1}\|f\|_{F} N_{j}(g) .
$$

At this stage we use the assumption that $(\Phi, K)$ is $F$-admissible to sanction the choice of $f_{i}^{*}(i=1,2, \cdots, j)$ from $F$ such that $f_{i}^{*}=0$ on some open subset $V$ of $G$ containing $K$ and

$$
2 c v j\left\|f_{i}^{*}-f_{i}\right\|_{F} \leqq \delta \quad(i=1,2, \cdots, j) .
$$

For $g \in E$ we then have:

$$
\begin{aligned}
N_{j}(g) & =\|g\|_{X}+\sum_{i=1}^{j}\left\|f_{i} g\right\|_{Z} \quad \text { (by 2.2) } \\
& \leqq\|g\|_{X}+\sum_{i=1}^{j}\left(\left\|f_{i}^{*} g\right\|_{Z}+\left\|\left(f_{i}^{*}-f_{i}\right) g\right\|_{Z}\right) \\
& \leqq\|g\|_{X}+\sum_{i=1}^{j}\left\|f_{i}^{*} g\right\|_{Z}+\sum_{i=1}^{j} c v \delta^{-1}\left\|f_{i}^{*}-f_{i}\right\|_{F} N_{j}(g),
\end{aligned}
$$

the last step by $(2.5)$, so that $(2.6)$ yields

$$
N_{j}(g) \leqq 2\left(\|g\|_{X}+\sum_{i=1}^{j}\left\|f_{i}^{*} g\right\|_{z}\right)
$$

for every $g \in E$. In particular, since $f_{i}^{*}=0$ on $V$ for $i=1,2, \cdots, j$, another use of (2.4) shows that 


$$
\|g\|_{Y} \leqq 2 v \delta^{-1}\|g\|_{X} \quad \text { if } g \in E, \operatorname{supp} g \subset V .
$$

A contradiction can now be reached on the basis of 1.3 in the following way. Take $U$ to be the nonvoid open subset $V K$ of $G$ and choose $g \in X_{U} \backslash Y$ and $g_{r} \in Y_{U}(r=1,2, \cdots)$ in accord with (1.3) and (1.4). Then $g_{r} \in E$ for every $r$ (since $g_{r} \in Y \subset X$ and $g_{r}$ vanishes on $K$; see 1.2 and (2.1)); since also supp $g_{r} \subset V$ for every $r,(2.7)$ applies with $g_{r}$ in place of $g$ to give

$$
\left\|g_{r}\right\|_{X} \leqq 2 v \delta^{-1}\left\|g_{r}\right\|_{X} \leqq 2 v \delta^{-1} m,
$$

that is, $g_{r} \in 2 v \delta^{-1} B$. But then (1.1) and (1.3) show that $g \in 2 v \delta^{-1} B \subset Y$, contrary to the choice of $g \in X_{U} \backslash Y$. This completes the proof.

2.4 CONCERNING CONDITION 1.3. In all the examples we consider the following conditions prevail:

(a) each of $X$ and $Y$ is translation invariant, and $X$ is stable under multiplication by functions belonging to a set $M$ (either $A(G)$ or $C^{\infty}(T)$, where $C^{\infty}(T)$ is defined as in $\left[3_{\mathrm{I}}\right]$, p. 27 and $\left[3_{\mathrm{II}}\right]$, Chapter 12$)$ which contains arbitrarily fine finite partitions of unity;

(b) there exist nonnegative continuous functions $v_{r} \neq 0$ on $G$ with supports contained in arbitrarily small neighbourhoods of zero in $G$ and such that $v_{r} * g \in Y, \sup _{r}\left\|v_{r} * g\right\|_{X}<\infty$, and $\mathscr{T}-\lim v_{r} * g=g$ whenever $g \in X$.

When these conditions are fulfilled, condition 1.3 is satisfied whenever $Y \neq X$.

For, if $Y \neq X$ and if (a) holds, a simple partition of unity argument shows that to any nonvoid open subset $U$ of $G$ corresponds $g \in X_{U} \backslash Y$. We then take $g_{r}$ to be a suitable subsequence of $v_{r} * g$, where the $v_{r}$ are as in (b) and have supports so small that supp $g_{r} \subset U$ for every $r$.

2.5 CONCERNING F-ADMisSibiLITY. We aim to give here some nontrivial sufficient conditions for $F$-admissibility in cases covering the examples discussed in §3. In many cases best-possible results are not known, but this question is not vital here since interesting applications result without need for extremely precise results about $F$-admissibility.

In all that follows, $\lambda$ will denote normalised Haar measure on the underlying group $G$ in question.

2.5.1 We begin with the case in which $G=T$, this being richer in examples. In the examples we shall consider, the following additional assumptions concerning $F$ will be satisfied:

(a) $C^{\infty} \subset F$ with a continuous injection;

(b) writing $e_{n}$ for the function $x \rightarrow e^{i n x}$ with domain $T,\left\|e_{n}\right\|_{F}=0\left(|n|^{\alpha}\right)$ for $n \in I,|n| \rightarrow \infty$. 
Introduce $F_{0}$ as the set of $f \in F$ such that, for every $l \in F^{\prime}$ (the dual of the normed linear space $F$ ),

$$
l(f)=\sum_{n \in I} l\left(e_{n}\right) \hat{f}(n),
$$

the series being absolutely convergent. It is plain that (a) and (b) guarantee that $C^{\infty} \subset F_{0}$.

2.5.2 Suppose that $F$ is as in 1.1 and 2.5.1. Let $K$ be a closed subset of $T$ different from $\emptyset$ and $T$, and let $\Phi$ be a nonvoid subset of $F$. Write $V_{j}(j=1,2, \cdots)$ for the interval $\left(-j^{-1}, j^{-1}\right)$ modulo $2 \pi$ and $D_{j}=\left(K+V_{j}\right) \backslash K$. In order that $(\Phi, K)$ be $F$-admissible, it is sufficient that

(a) $\Phi \subset F_{0}$ and $K \subset Z(\Phi)$, and

(b) $\lim _{j \rightarrow \infty} j^{2 x+1} \int_{D_{j}}|f|^{2} d \lambda=0 \quad$ for every $f \in \Phi$.

Since $\lambda\left(D_{j}\right) \rightarrow 0$ as $j \rightarrow \infty$, (b) will be satisfied if each $f \in \Phi$ is such that $f(x)=$ $0\left(d^{\kappa+\frac{1}{2}}\right)$ for every $x$ at distance at most $d$ from $K$.

Proof. By the Hahn-Banach theorem, we need to show that, if $f \in \Phi$ and if a continuous linear functional $l$ on $F$ annihilates every element of $F$ which vanishes on some neighbourhood of $K$, then $l(f)=0$. In view of 2.5.1(a) and 2.3.1 of [6], there is a distribution $S$ on $T$ such that $S\left(e_{n}\right)=l\left(e_{n}\right)$ for every $n \in I$, and (2.5.1) shows that

$$
l(f)=S(f)=\sum_{n \in I} l\left(e_{n}\right) \hat{f}(n)
$$

for every $f \in C^{\infty}$. Moreover, by $2.5 .1(\mathrm{~b})$,

$$
\left|l\left(e_{n}\right)\right| \leqq c(1+|n|)^{\kappa} \quad(n \in I)
$$

for some real number $c \geqq 0$.

The fact that $l$ annihilates every element of $F$ which vanishes on a neighbourhood of $K$ combines with (2.5.2) to show that supp $S \subset K$. Take an approximate identity for convolution formed of functions $k_{j} \in C^{\infty}$ such that supp $k_{j} \subset V_{j}$. Then $k_{j} * f \in C^{\infty}$ and (2.5.2) gives

$$
\begin{aligned}
l\left(k_{j} * f\right)=S\left(k_{j} * f\right) & =\left(\check{k}_{j} * S\right)(f) \\
& =\int S_{j} f d \lambda,
\end{aligned}
$$

where $S_{j}=\check{k}_{j} * S \in C^{\infty}$, so that supp $S_{j} \subset K+V_{j}$, and $\check{k}_{j}(x)=k_{j}(-x)$. On the other hand, $\lim _{j \rightarrow \infty} \hat{k}_{j}=1$ boundedly on $I$ and so, since 2.5.2(a) guarantees that $f \in F_{0}$, the assumed absolute convergence of the series appearing in (2.5.1) ensures that 


$$
\begin{aligned}
l(f)=\sum_{n \in I} l\left(e_{n}, \hat{f}(n)\right. & =\lim _{j \rightarrow \infty} \sum_{n \in I} l\left(e_{n}\right)\left(k_{j} * f\right)(n) \\
& =\lim _{j \rightarrow \infty} l\left(k_{j} * f\right) \\
& =\lim _{j \rightarrow \infty} \int S_{j} f d \lambda .
\end{aligned}
$$

Thus, since supp $S_{j} \subset K+V_{j}$ and $f$ vanishes on $K$ (by 2.5.2(a)),

$$
l(f)=\lim _{j \rightarrow \infty} \int_{D_{j}} S_{j} f d \lambda .
$$

By the Parseval formula, the Cauchy-Schwarz inequality, (2.5.3) and (2.5.4), we have

$$
|l(f)| \leqq \text { const. } \lim \inf _{j \rightarrow \infty}\left\{w_{j} \int_{D_{j}}|f|^{2} d \lambda\right\}^{\frac{1}{2}},
$$

where

$$
w_{j}=\sum_{n \in I}(1+|n|)^{2 \kappa}\left|\hat{k}_{j}(n)\right|^{2} .
$$

Straightforward calculation confirms that one may choose the $k_{j}$ so that $w_{j}=$ $0\left(j^{2 k+1}\right)$ for large $j$, so that $(2.5 .5)$ and 2.5.2(b) lead to the conclusion $l(f)=0$, as required.

2.5.3 In case of a more general group $G$ (see 1.1 above), the substance of 2.5.1 and 2.5.2 can be modified by replacing both $C^{\infty}$ and $F_{0}$ by $A(G)$ and formally taking $\kappa=0$; one can then adapt the arguments in 13.5 .5 of $\left[3_{\text {II }}\right]$ in such a way as to reach the following conclusion.

Suppose that $A(G) \subset F$ with a continuous injection and that

$$
\sup _{\gamma \in \Gamma}\|\gamma\|_{F}<\infty
$$

where $\Gamma$ denotes the group dual to $G$. Suppose that $\Phi$ is a nonvoid subset of $A(G)$ and $K$ a nonopen closed subset of $G$ contained in $Z(\Phi)$. In order that $(\Phi, K)$ be $F$-admissible, it is sufficient that either

(a) $K$ is a spectral synthesis set (see $\left[3_{\text {II }}\right], 12.11 .4$ ), or

(b) to each $f \in \Phi$ corresponds a base $\left(V_{j}\right)_{j=1}^{\infty}$ of neighbourhoods of zero in $G$ such that

where

$$
\lim _{j \rightarrow \infty} \lambda\left(V_{j}\right)^{-1} \int_{D_{j}}|f|^{2} d \lambda=0
$$

$$
D_{j}=\left(K+V_{j}\right) \backslash K
$$

Condition (b) will be satisfied if each $f \in \Phi$ satisfies 
for large $j$.

$$
\sup _{x \in D_{j}}|f(x)|=O\left(\lambda\left(V_{j}\right)^{\frac{1}{2}}\right)
$$

Note that $K$ is a spectral synthesis set whenever its frontier contains no nonvoid perfect set (cf. [3 $3_{I I}$, Exercise 12.52).

\section{Applications}

3.1 Notations AND PReliminary Remarks. In this subsection we collect together some of the notations and preliminary remarks used throughout the examples to follow.

$G$ and $C(G)$ are as described in 1.1 and, as in $2.5, \lambda$ denotes normalised Haar measure on $G$. $\Gamma$ denotes the group dual to $G$. For $1 \leqq p \leqq \infty, L^{p}(G)$ denotes the usual Lebesque space constructed relative to $\lambda$ and $l^{p}(\Gamma)$ the similar space constructed relative to counting measure on $\Gamma ;\|\cdot\|_{p}$ denotes the corresponding norm on $L^{p}(G)$ or on $l^{p}(\Gamma)$, the context making plain which is involved.

For $f \in L^{1}(G), \hat{f}$ denotes the Fourier transform of $f$ :

$$
f(\gamma)=\int_{G} f(x) \overline{\gamma(x)} d \lambda(x) \quad(\gamma \in \Gamma) .
$$

For any number $p$ satisfying $1 \leqq p \leqq \infty, p^{\prime}$ will always denote the conjugate exponent defined by $p^{\prime}=p /(p-1)$.

3.1.1 If $1 \leqq r \leqq \infty, A^{r}(G)$ will denote the set of $f \in C(G)$ such that $\hat{f} \in I^{r}(I)$, regarded as a Banach space with the norm

$$
\|f\|_{A^{r}(G)}=\max \left\{\|f\|_{\infty},\|\hat{f}\|_{r}\right\} .
$$

When $r=1$ we usually write $A(G)$ in place of $A^{1}(G)$; this is the direct extension (from $T$ to $G$ ) of the space $A$ mentioned in $\S 0$. If $r \geqq 2, A^{r}(G)=C(G)$; and in all cases $A(G) \subset A^{r}(G)$ with a continuous injection.

By using summability factors (similar to those described in Theorem (3.4) of [5]) it can be shown that, if $1 \leqq q \leqq \infty, f \in L^{q}(G)$ and $g \in L^{q^{\prime}}(G)$, then

$$
\left|(f g)^{\wedge}(\gamma)\right| \leqq|\hat{f}| *|\hat{g}|(\gamma)
$$

for every $\gamma \in \Gamma$; an application of Young's inequality for the group $\Gamma$ (cf. $\left[3_{\mathrm{II}}\right]$, p. 150) then shows that

$$
\left\|(f g)^{\wedge}\right\|_{t} \leqq\|\hat{f}\|_{r}\|\hat{g}\|_{s}
$$

whenever

$$
1 \leqq s \leqq \infty, \quad 1 \leqq r \leqq s^{\prime}, \quad 1 / t=1 / r+1 / s-1 .
$$

3.1.2 In 3.1.3-3.1.5 we specialise by supposing that $G=T$, so that $\Gamma$ becomes the additive group $I$ of integers. Whenever this is the case, we write $C, A^{r}, L^{p}, \cdots$ 
in place of $C(G), A^{r}(G), L^{p}(G), \cdots$ and $l^{r}$ in place of $l^{r}(I)$. Also, we shall continue to write $e_{n}$ for the function $x \rightarrow e^{i n x}$ on $T$ whenever $n \in I$; and

$$
s_{N} f=\sum_{|n| \leqq N} \hat{f}(n) e_{n}
$$

will denote the $N$-th symmetric partial sum of the Fourier series of $f \in L^{1}, N$ denoting a nonnegative integer.

3.1.3 For $\alpha \in(0,1], \delta>0$ and $f \in C$ we write

and

$$
M_{\alpha, \delta}(f)=\sup \left\{\delta^{-\alpha}|f(x)-f(y)|: x, y \in T,|x-y| \leqq \delta\right\}
$$

$$
M_{\alpha}(f)=\sup _{\delta>0} M_{\alpha, \delta}(f)
$$

and define $\Lambda_{\alpha}$ to be the set of $f \in C$ for which $M_{\alpha}(f)$ is finite. Endowed with the norm

$$
\|f\|_{A_{\alpha}}=\|f\|_{\infty}+M_{\alpha}(f),
$$

$\Lambda_{x}$ is a Banach space.

The set of $f \in \Lambda_{\alpha}$ for which

$$
\lim _{\delta \rightarrow 0} M_{\alpha, \delta}(f)=0
$$

is a closed linear subspace of $\Lambda_{\alpha}$ which we denote by $\lambda_{\alpha}$ and view as a Banach space with the induced norm.

Two facts concerning $\lambda_{\alpha}$ will be needed later.

(i) If $f \in \lambda_{\alpha}$, then $\left\|T_{a} f-f\right\|_{\Lambda_{\alpha}} \rightarrow 0$ as $a \rightarrow 0$, where $T_{a}$ denotes the operator of translation by amount $a$; see, for example, [7], p. 16, Exercise 4 (where $A_{\alpha}$ is denoted by $\operatorname{Lip} \alpha$ and $\lambda_{\alpha}$ by $\operatorname{lip} \alpha$ ). From this it follows that

$$
\lim _{r \rightarrow \infty}\left\|v_{r} * f-f\right\|_{\Lambda_{\alpha}}=0
$$

whenever $f \in \lambda_{\alpha}$ and $\left(v_{r}\right)$ is an approximate identity for convolution (which may be extracted from $C^{\infty}(T)$ if one wishes).

(ii) If $1 \leqq s<2$ and $\alpha=1 / s-\frac{1}{2}$, then

$$
\lambda_{\alpha} \notin A^{s} \text {. }
$$

In fact, it is known ([4], p. 243) that, if $\alpha$ and $s$ are as specified, then

$$
\Lambda_{\alpha} \neq A^{s} \text {. }
$$

Where (3.1.4) to be false, the closed graph theorem ([6], Theorem 6.4.2) would show that

$$
\|\hat{f}\|_{s} \leqq \text { const. }\|f\|_{\Lambda_{\alpha}}
$$

for every $f \in \lambda_{\alpha}$. But then, if $g \in \Lambda_{\alpha}$ and $\left(v_{r}\right)$ is an approximate identity extracted 
from $C^{\infty}(T)$, we should have $\left\|v_{r} * g\right\|_{\Lambda_{\alpha}}$ bounded with respect to $r$, so that (3.1.6) applied with $f=v_{r} * g$ would show that $\left\|\hat{v}_{r} \hat{g}\right\|_{s}$ would be bounded with respect to $r$ and hence that $g \in A^{s}$. This conflict with (3.1.5) provides an indirect proof of (3.1.4).

3.1.4 We denote by $A C$ the Banach space of absolutely continuous functions on $T$, the norm being

$$
\|f\|_{A C}=\|f\|_{\infty}+\|D f\|_{1},
$$

wherein $D$ denotes derivation.

Also, if $1 \leqq r \leqq \infty, A C^{r}$ will denote $A^{r} \cap A C$, made into a Banach space with the norm

$$
\|f\|_{A C r}=\max \left\{\|f\|_{A C},\|\hat{f}\|_{r}\right\} .
$$

If $r>1, A C^{r}$ and $A C$ are identical as linear spaces and their norms are equivalent.

3.1.5 We denote by $C_{*}$ the set of $f \in C$ having uniformly bounded Fourier partial sums (UBFPS for short), i.e., for which

$$
\|f\|_{*}=\sup \left\{\left\|s_{N} f\right\|_{\infty}: N=0,1,2, \cdots\right\}
$$

is finite. It is easily seen that

$$
\|f\|_{\infty} \leqq\|f\|_{*},
$$

from which it follows that $C_{*}$ is a Banach space and that the injection of $C_{*}$ into $C$ is continuous.

3.2 Application to $A^{s}(G)$. In our first application we suppose that

$$
1 \leqq s<2, \quad 1 \leqq r \leqq s^{\prime}, \quad 1 / t=1 / r+1 / s-1
$$

and aim to apply Theorem 2.1 on choosing

$$
X=C(G), \quad Y=A^{s}(G), \quad Z=A^{t}(G), \quad F=A^{r}(G),
$$

the notation being as described in 3.1.2. For $\mathscr{T}$ we take the norm topology on $X$.

It is clear that all the assumptions of 1.1 hold, and that (1.1) is verified. The remainder of 1.2 is also guaranteed by (3.1.1).

We may apply 2.4 (with $M$ taken to be $A(G)$ ) and (3.1.1) to infer that 1.3 is satisfied on account of the fact that $Y \neq X$ (i.e., that there exist continuous functions $f$ on $G$ such that $\hat{f} \notin l^{s}(\Gamma)$ ): this is the case since $G$ is infinite and $s<2$ (cf., for example, [ $\left.3_{\mathrm{II}}\right], 15.3 .2$ and 15.2.5).

Concerning $A^{r}(G)$-admissibility, see 2.5.3.

Appeal to 2.1 yields the following conclusion:

3.2.1 If $r, s$ and $t$ are as in (3.2.1) and if $(\Phi, K)$ is a countable $A^{r}(G)$-admissible pair, there exist continuous functions $g$ on $G$ such that $g=0$ on $K$, $(f g)^{\wedge} \in l^{t}(\Gamma)$ for every $f \in \Phi$, and yet $\hat{g} \notin l^{s}(\Gamma)$. 
In the next two examples we take $G=T$ and strengthen the conclusion of 3.2.1 in certain respects.

3.3 Application to $\lambda_{\alpha}$. We take $s, r, t, F$ and $Z$ as in 3.2. But now, supposing $G$ to be the circle group $T$, we take for $X$ the space $\lambda_{\alpha}$ defined in 3.1.3 with $a=1 / s-\frac{1}{2}$, for $\mathscr{T}$ the norm topology on $X$, and for $Y$ the space $\lambda_{\alpha} \cap A^{s}$ with norm

$$
\|f\|_{Y}=\max \left\{\|f\|_{X},\|\hat{f}\|_{s}\right\} .
$$

The verification of 1.1 and 1.2 proceeds as in 3.2. On the other hand, 1.3 is now equivalent (via 2.4) to (3.1.4).

An application of 2.1 yields the following refinement of 3.2.1.

3.3.1 Suppose that $r, s, t$ are as in (3.2.1) and that $\alpha=1 / s-\frac{1}{2}$, and let $(\Phi, K)$ be a countable $A^{r}$-admissible pair. Then there exist functions $g \in \lambda_{\alpha}$ such that $g=0$ on $K,(f g)^{\wedge} \in l^{t}$ for every $f \in \Phi$, and yet $\hat{g} \notin l^{s}$.

3.3.2 In view of the substance of 2.5.2 (with $F=F_{0}=A$ and $\kappa=0$ ) or 2.5.3, the case $r=s=t=1$ of 3.3.1 yields 0.3 .

3.4 Application to $A C^{1}$. Here we take (using the notation described in 3.1.4) $X=A C$ and $\mathscr{T}$ the norm topology on $X, F=Z=A C^{r}$ and $Y=A C^{1}$.

It is again easy to verify that 1.1 and 1.2 are fulfilled. Moreover, in view of $2.4,1.3$ is equivalent to the existence of functions $g \in A C$ such that $\hat{g} \notin l^{1}$, concerning which see $\left[3_{\mathrm{I}}\right], 10.6 .1(2)$.

From 2.1 we accordingly derive the following conclusion.

3.4.1 If $1 \leqq r \leqq \infty$ and $(\Phi, K)$ is a countable $A C^{r}$-admissible pair, there exist absolutely continuous functions $g$ such that $g=0$ on $K,(f g)^{\wedge} \in l^{r}$ for every $f \in \Phi$, and yet $\hat{g} \notin l^{1}$.

3.4.2 Concerning $A C^{r}$-admissibility, 2.5.2 applies with $\kappa=1$; thus 2.5.2(b) will be satisfied provided $f(x)=O\left(d^{\frac{3}{2}}\right)$ for all $x$ at distance at most $d$ from $K$ (which is so, provided $f \in C^{1}$ and $f=D f=0$ on $K$ ).

3.4.3 We cannot hope to 'combine' 3.3 and 3.4 to the extent of choosing $g$ so as to belong simultaneously to $\Lambda_{\alpha}(\alpha>0)$ and to $A C$, since $\Lambda_{\alpha} \cap A C \subset A$ ([3] $]$, p. 167).

3.5 Application to conjugate functions. Here again we take $G=T$ and denote by $f^{\sim}=H * f$ the function conjugate to $f$ (cf. [3 $3_{\text {II }}$, pp. 86 et seq.).

3.5.1 It is our intention to apply Theorem 2.1 , taking $X=A C$ and $\mathscr{T}$ as the norm topology on $X$;

with norm

$$
Z=Y=\left\{f \in A C: f^{\sim} \in L^{\infty}\right\}
$$


and

$$
\|f\|_{Z}=\|f\|_{Y}=\|f\|_{A C}+\|\hat{f}\|_{\infty}
$$

$$
F=\left\{f \in A C: \sum_{n \in I}|n|^{\frac{1}{2}}|\hat{f}(n)|<\infty\right\}
$$

with norm

$$
\|f\|_{F}=\|f\|_{A C}+\sum_{n \in I}(1+|n|)^{\frac{1}{2}}|\hat{f}(n)| .
$$

It is plain that all this conforms with 1.1.

3.5.2 Concerning 1.2, we first note that $f_{k} \rightarrow f$ in $X$ ensures that $f_{k}^{\sim} \rightarrow f^{\sim}$ in $L^{2}$, which makes it easy to verify that $B$ is a $\mathscr{T}$-closed subset of $X$.

Next we have the identity

$$
\left(e_{k} g\right)^{\sim}-e_{k} g^{\sim}=e_{k} \sum_{n \in I}\{\operatorname{sgn}(n+k)-\operatorname{sgn}(n)\} \hat{g}(n) e_{n},
$$

the right hand side being a trigonometric polynomial. The Cauchy-Schwarz inequality and the Parseval formula lead from (3.5.3) to

$$
\left\|\left(e_{k} g\right)^{\sim}-e_{k} g^{\sim}\right\|_{\infty} \leqq|k|^{\frac{1}{2}}\|g\|_{2}
$$

and thence to the inequality

$$
\left\|\left(\left(s_{N} f\right) g\right)^{\sim}-\left(s_{N} f\right) g^{\sim}\right\|_{\infty} \leqq\|g\|_{\mid} \sum_{|n| \leqq N}|n|^{\frac{1}{1}}|\hat{f}(n)| .
$$

If $f \in F$ and $g \in Y, s_{N} f \rightarrow f$ uniformly as $N \rightarrow \infty$ and so $\left(\left(s_{N} f\right) g\right)^{\sim} \rightarrow(f g)^{\sim}$ in $L^{2}$. In combination with (3.5.1) and (3.5.4), this shows that $(f g)^{\sim} \in L^{\infty}$ and that

$$
\left\|(f g)^{\sim}\right\|_{\infty} \leqq\|f\|_{\infty}\left\|g^{\sim}\right\|_{\infty}+\|f\|_{F}\|g\|_{2} .
$$

Since also $f g \in A C$, it is now easy te verify that $F Y \subset Z$ and that $\left(1.2^{\prime}\right)$ holds with a suitable $c$.

3.5.3 As to 1.3 , it is known $\left(\left[3_{\mathrm{II}}\right]\right.$, p. 91$)$ that there exist functions $g \in A C$ such that $g^{\sim} \notin L^{\infty}$, so that $X \neq Y$. On the basis of 2.4 we can therefore be sure that 1.3 is satisfied.

At this point we may apply Theorem 2.1 to derive the following conclusion.

3.5.4 Suppose that $F$ is as described in (3.5.1) and (3.5.2) and that $(\Phi, K)$ is a countable $F$-admissible pair. Then there exist functions $g \in A C$ such that $g=0$ on $K, f g \in A C$ and $(f g)^{\sim} \in L^{\infty}$ for every $f \in \Phi$, and $g^{\sim} \notin L^{\infty}$.

3.5.5 Regarding $F$-admissibility, we may revert to 2.5 .2 for sufficient conditions. In the present case we may take $\kappa=1$, so that $F_{0}$ contains at least all $f \in C$ such that

$$
\sum_{n \in I}|n \hat{f}(n)|<\infty .
$$

If $\Phi$ contains only such functions, and if $K$ is a closed subset of $T$ different from 
$\emptyset$ and $T$ and contained in $Z(\Phi)$, then $(\Phi, K)$ is $F$-admissible provided

$$
\lim _{j \rightarrow \infty} j^{3} \int_{D_{j}}|f|^{2} d \lambda=0
$$

for every $f \in \Phi$. As in 3.4.2, this limit relation is satisfied provided every $f \in \Phi$ satisfies $f(x)=0\left(d^{\frac{3}{2}}\right)$ for every $x$ at distance $\leqq d$ from $K$ (and so whenever every $f \in \Phi$ satisfies $f=D f=0$ on $K$ ).

3.6 APPLICATION TO CONTINUOUS FUNCTIONS WITH UNIFORMLY BOUNDED FOURIER PARTIAL SUMS

3.6.1 We now take $X=C$ with $\mathscr{T}$ the norm topology on $X$ and define both $Y$ and $Z$ to be the space $C_{*}$ defined in 3.1.5.

For $F$ we take the space of functions $f \in C$ for which

$$
\|f\|_{F}=\sum_{n \in I}(1+|n|)^{\frac{1}{2}}|\hat{f}(n)|
$$

is finite. Again it is clear that the injection of $F$ into $C$ is continuous.

The conditions of 1.1 are thus met.

3.6.2 Regarding 1.2, it is first of all clear that (1.1) holds. Moreover, it may be verified (cf. 3.5.2) that $e_{k} g \in Z$ and

$$
\left\|e_{k} g\right\|_{z} \leqq \text { const. }(1+|k|)^{\frac{1}{2}}\|g\|_{Z}
$$

whenever $g \in Y$ and $k \in I$. Using the completeness of $Z$ and the continuity of the injection of $Z$ into $C$, it then follows that $F \cdot Y \subset Z$ and that

$$
\|f g\|_{Z} \leqq \text { const. }\|f\|_{F}\|g\|_{Z}
$$

whenever $f \in F$ and $g \in Y$. Thus (1.2) and (1.2') are satisfied.

3.6.3 The verification of 1.3 proceeds by reference to 2.4 , taking $M=C^{\infty}$. (That $M \cdot Y \subset Y$ follows from the substance of 3.6.2.)

All the hypotheses stated in $\S 1$ being satisfied, an appeal to Theorem 2.1 leads to the following conclusion.

3.6.4 If $(\Phi, K)$ is a countable $F$-admissible pair, with $F$ being as described in 3.6.1, there exist continuous functions $g$ vanishing on $K$, such that $f g$ has UBFPS for every $f \in \Phi$, and yet $g$ has not UBFPS.

3.6.5 Conditions sufficient to ensure $F$-admissibility stem from 2.5.2. In the present case we may take $\kappa=\frac{1}{2}$ and $F_{0}=F$. The relation 2.5.2(b) is accordingly satisfied whenever $\Phi \subset A_{1}$ (defined as in 3.1.3); or whenever $K$ is a finite union of points and intervals (in which case $\lambda\left(D_{j}\right)=0\left(j^{-1}\right)$ ) and every $f \in \Phi$ belongs to $\Lambda_{\alpha}$ for some (possibly $f$-dependent) $\alpha>\frac{1}{2}$. 
3.7 APPLICATION TO CONTINUOUS FUNCTIONS WITH UNIFORMLY CONVERGENT FOURIER SERIES

3.7.1 We here take for $X$ the space $C_{*}$. For $\mathscr{T}$ we take the topology of uniform convergence (induced on $X$ by the topology of $C$ ).

Let $\left(\varepsilon_{N}\right)_{N=0}^{\infty}$ be a sequence of positive numbers. The interesting cases are those in which $\lim _{N \rightarrow \infty} \varepsilon_{N}=0$, but we do not need to assume this to be the case from the outset. For $Y$ we choose the linear subspace of $g \in X$ such that

$$
\left\|s_{N} g-g\right\|_{\infty}=O\left(\varepsilon_{N}\right) \quad(N=0,1,2, \cdots),
$$

the norm on $Y$ being defined by

$$
\|g\|_{Y}=\|g\|_{*}+\sup _{N} \varepsilon_{N}^{-1}\left\|g-s_{N} g\right\|_{\infty} .
$$

For $Z$ we take the set of $g \in X$ such that $g$ has a uniformly convergent Fourier series (UCFS for short), the norm on $Z$ being the restriction to $Z$ of that on $C_{*}$.

$F$ is defined in 3.6.1.

All the conditions in 1.1 are then satisfied.

3.7.2 Turning to 1.2 , it is simple to verify that $B$ is $\mathscr{T}$-closed in $X$.

The verification of (1.2) is less immediate. We note first that (3.6.2) may be expressed in the current notation by the assertion

$$
F \cdot X \subset X \text { and }\|f g\|_{X} \leqq \text { const. }\|f\|_{F}\|g\|_{X} \quad(f \in F, g \in X) .
$$

Also, as is quite clear,

$$
f g \in Z \text { whenever } f \in F \text { and } g \text { is a trigonometric polynomial. }
$$

As a final preliminary, note that

$$
Z \text { is norm closed in } X \text {. }
$$

Now suppose that $g \in Y$; then $s_{N}\left(g-s_{q} g\right)$ is 0 if $N \leqq q$ and is $s_{N} g-s_{q} g$ if $N>q$, so that

$$
\left\|g-s_{q} g\right\|_{X} \leqq \sup _{N>q}\left\|s_{N} g-s_{q} g\right\|_{\infty},
$$

which tends to 0 as $q \rightarrow \infty$ since the Fourier series of $g$ is uniformly convergent. Thus

$$
\lim _{q \rightarrow \infty}\left\|g-s_{q} g\right\|_{X}=0 \quad(g \in Y) .
$$

Therefore, if $f \in F$ and $g \in Y$, (3.7.3) and (3.7.6) show that

$$
\lim _{q \rightarrow \infty}\left\|f g-f \cdot s_{q} g\right\|_{X}=0 ;
$$

this, together with (3.7.4) and (3.7.5), entails that $f g \in Z$. Moreover, by (3.7.3) and (3.7.7), 


$$
\begin{aligned}
\|f g\|_{Z}=\|f g\|_{X} & =\lim _{q \rightarrow \infty}\left\|f \cdot s_{q} g\right\|_{X} \\
& \leqq \text { const. }\|f\|_{F} \cdot \lim _{q \rightarrow \infty}\left\|s_{q} g\right\|_{X} \\
& =\text { const. }\|f\|_{F}\|g\|_{X},
\end{aligned}
$$

the last step by (3.7.6). In particular, reference to (3.7.2) and (3.7.3) now shows that $F \cdot Y \subset Z$ and $\|f g\|_{Z} \leqq$ const. $\|f\|_{F}\|g\|_{Y}$ for $f \in F$ and $g \in Y$. This verifies (1.2) and $\left(1.2^{\prime}\right)$.

3.7.3 All the conditions of 2.4 are met: (a) follows with $M=C^{\infty}$ from the substance of 3.7.2; and (b) is satisfied if we take $v_{r} \in C^{\infty}$ forming an approximate identity for convolution with supp $v_{r} \downarrow\{0\}$. Therefore, 1.3 will be satisfied whenever $Y \neq X$.

Now reference to pp. $125-127$ of $\left[2_{I}\right]$ shows that $Y$ is indeed distinct from $X$ provided there is an integer $a$ such that

$$
a>\exp (2 b \sqrt{ } 2)
$$

where

and

$$
b=\sup \left\{\left|\sum_{k=1}^{n} k^{-1} \sin k x\right|: n=1,2, \cdots, x \text { real }\right\}
$$

$$
\underset{r \rightarrow \infty}{\liminf \varepsilon_{N_{r}}}=0, \quad \text { where } N_{r}=2 a^{r^{2}}-1
$$

This being so, we may infer from 2.1 the following result.

3.7.4 Suppose that $\left(\varepsilon_{N}\right)_{N=0}^{\infty}$ is a sequence of positive numbers satisfying (3.7.9) for some choice of the positive integer $a$ satisfying (3.7.8). Suppose also that $F$ is as defined in 3.6.1 and that $(\Phi, K)$ is a countable $F$-admissible pair (cf. 3.6.5). Then there exist continuous functions $g$ with UBFPS which vanish on $K$, which are such that $f g$ has a UCFS for every $f \in \Phi$, and yet which satisfy

$$
\limsup _{N \rightarrow \infty} \varepsilon_{N}^{-1}\left\|g-s_{N} g\right\|_{\infty}=\infty \text {. }
$$

\section{Concerning 0.1}

4.1 In all the applications we have discussed our choice of $F$ has been in accord with the following stipulations:

(a) $F$ contains a set $F_{1}$ which is a subalgebra (with respect to pointwise operations) of $C(G)$, containing all constant functions and endowed with a norm (possibly different from that induced by the norm on $C(G)$ and from that on $F$ ) making it into a Banach algebra, the injection of $F_{1}$ into $C(G)$ being continuous;

(b) if $f \in F_{1}$, then the complex conjugate function $\bar{f} \in F_{1}$; 
(c) every nonzero continuous complex homomorphism of $F_{1}$ is of the type $f \leftrightarrow f(x)$ for some $x \in G$;

(d) if $L$ is any closed subset of $G$ and $x_{0} \in G \backslash L$, there exists $f_{0} \in F_{1}$ such that $f_{0}\left(x_{0}\right) \neq 0$ and $f_{0}(x)=0$ for every $x \in L$.

As we shall now show, this circumstance leads to a conclusion of which 0.1 is the special case $G=T, F_{1}=F=Z=A$.

4.2 Suppose that $F$ satisfies conditions (a)-(d) in 4.1, that $\Phi$ is a nonvoid subset of $F_{1}$, and that $U$ is any neighbourhood in $G$ of $Z(\Phi)$. Suppose further that $Z$ is a set of complex-valued functions on $G$ such that $Z+Z \subset Z$ and $F_{1} \cdot Z \subset Z$. If $g$ is any complex-valued function on $G$ such that $g=0$ on $U$ and $f g \in Z$ for every $f \in \Phi$, then $g \in Z$.

Proof. We may assume that $U$ is open in $G$. The argument proceeds in two stages, the first of which refers only to $F_{1}$.

(i) Consider the ideal $I$ in $F_{1}$ formed of those $f \in F_{1}$ which vanish on the closed subset $L=G \backslash U$ of $G$. The final clause of (a) ensures that $I$ is closed in $F_{1}$, so that $F_{1} / I$ is a commutative Banach algebra with identity. From (c) it follows that every nonzero continuous complex homomorphism of $F_{1} / I$ is of the type $f+I \mapsto f(x)$ for some $x \in G$, and (d) goes to show that $x$ must belong to $L=G \backslash U$. The Gelfand theory (specifically, 11.4 .10 of $\left[3_{\text {III }}\right]$ ) now shows that, if $f \in F_{1}$ is nonvanishing on $G \backslash U$, there exists $h \in F_{1}$ such that $h f=1$ on $G \backslash U$.

(ii) Since $G \backslash U$ is compact and $U \supset Z(\Phi)$, there exist $f_{1}, \cdots, f_{n} \in \Phi$ such that

$$
f=\sum_{i=1}^{n} f_{i} f_{i}
$$

is nonvanishing on $G \backslash U$. On the other hand, (a) and (b) show that $f$ is an element of $F_{1}$. By (i), therefore, there exists $h \in F_{1}$ such that

$$
h f=\sum_{i=1}^{n} h \bar{f}_{i} f_{i}=1 \quad \text { on } G \backslash U .
$$

Hence, since $g$ vanishes on $U$,

$$
g=\sum_{i=1}^{n}\left(h f_{i}\right)\left(f_{i} g\right)
$$

wherein $f_{i} g \in Z$ and $h f_{i} \in F_{1}$ (the first by hypothesis on $g$, and the second by 4.1(a) and (b)). The hypotheses on $Z$ then combine with (4.1) to show that $g \in Z$.

4.3 If $F$ has any one of the meanings described in 3.4-3.7 above, 4.1 is applicable with $F_{1}=F$. But if we choose $F=A^{r}$ (as in 3.2 and 3.3), and if $r \in(1,2)$, the situation is not clear: it appears to be a nontrivial open problem to determine whether $A^{r}$ is or is not an algebra under pointwise operations. (We may, of course, apply 4.1 with $F_{1}=A$.) 


\section{A constructive approach when $G=T$}

5.1 It is assumed throughout this section that $G=T$ and that $\Phi$ is a nonvoid subset of $C$ with the following properties:

(1) to each $f \in \Phi$ corresponds $p=p_{f}>1$ such that $D f \in L^{p}$;

(2) $Z(\Phi)$ is nonvoid.

Our object is to indicate an explicit construction leading to functions $g \in A C$ such that

(3) $f g \in A$ for every $f \in \Phi$;

(4) $g \notin A$.

The outcome bears comparison with the case $G=T, r=1$ of 3.4.1. Condition (1) immediately above entails ([3 $\left.3_{\mathrm{II}}\right]$, Exercise 13.2) that, as in 3.4.1, $\Phi$ is a subset of $A C^{1}$. On the other hand, we do not here assume that $\Phi$ is countable; and we do not here assert anything about $g$ vanishing on subsets $K$ of $Z(\Phi)$ ? so that admissibility considerations no longer arìse).

5.2 Construction of $g$. We start with any function $\phi \in L^{\infty}$ such that

$$
D \phi=\mu+\psi,
$$

where $\mu$ is a measure on $T$ satisfying

$$
\operatorname{supp} \mu \subset Z(\Phi), \sum_{n \neq 0}\left|n^{-1} \hat{\mu}(n)\right|=\infty
$$

and

$$
\psi \in L^{q} \text { for some } q>1 .
$$

A possible choice is to take $a \in Z(\Phi)$ and

$$
\phi(x)=\sum_{n \neq 0}(i n)^{-1} e^{i n(x-a)},
$$

corresponding to which $\mu=\varepsilon_{a}$ (the Dirac measure placed at $a$ ) and $\psi=1$.

Then, by [ $\left.3_{11}\right]$, Exercise 13.1 and Hölder's inequality,

$$
\sum_{n \neq 0}\left|n^{-1} \hat{\psi}(n)\right|<\infty,
$$

so that (5.2.1) and (5.2.2) arrange that

$$
n \hat{\phi}(n)=O(1), \sum_{n \in I}|\hat{\phi}(n)|=\infty .
$$

Thanks to (5.2.5), a sequence $\left(\lambda_{N}\right)_{N=1}^{\infty}$ can be constructed so that

$$
\lambda_{N}>0, \sum_{N=1}^{\infty} \lambda_{N}<\infty
$$




$$
\sum_{n \in I}|\hat{\phi}(n)| \sum_{N=1}^{\infty} \lambda_{N} \hat{F}_{N}(n)=\infty
$$

where $F_{N}$ denotes Fejér's kernel.

Now define

$$
g=\sum_{N=1}^{\infty} \lambda_{N}\left(F_{N} * \phi\right)=h * \phi
$$

where

$$
h=\sum_{N=1}^{\infty} \lambda_{N} F_{N} .
$$

Then (5.2.6) combines with the fact that $\left\|F_{N}\right\|_{1}=1$ to show that $h \in L^{1}$, so that $g \in C$. What is more, (5.2.1)-(5.2.3) and (5.2.8) show that

$$
D g=h * D \phi=h * \mu+h * \psi \in L^{1},
$$

so that $g \in A C$. Furthermore, (5.2.8) yields

$$
\hat{g}(n)=\hat{\phi}(n) \sum_{N=1}^{\infty} \lambda_{N} \widehat{F}_{N}(n),
$$

and (5.2.7) guarantees that $g \notin A$.

It remains to show that $f g \in A$ whenever $f \in \Phi$. Now $D(f g)=D f \cdot g+f \cdot D g$, of which the first summand belongs to $L^{p}$ by 5.1(1). On the other hand,

$$
f \cdot D g=f \cdot(h * \mu)+f \cdot(h * \psi),
$$

of which the second summand belongs to $L^{q}$ in view of (5.2.3) and the fact that $h \in L^{1}$. It will therefore suffice ([3 $\left.3_{\mathrm{II}}\right]$, Exercise 13.2) to show that

$$
f \cdot(h * \mu) \in L^{s} \quad \text { for some } s>1 .
$$

But, by (5.2.8) and Fatou's lemma,

$$
\|f \cdot(h * \mu)\|_{s} \leqq \sum_{N=1}^{\infty} \lambda_{N}\left\|f \cdot\left(F_{N} * \mu\right)\right\|_{s}
$$

and so (5.2.6) will ensure (5.2.10), provided

$$
\sup _{N}\left\|f \cdot\left(F_{N} * \mu\right)\right\|_{s}<\infty \quad \text { for some } s>1 .
$$

An appeal to Hölder's inequality and the Fubini theorem leads to the estimate

$$
\left\|f \cdot\left(F_{N} * \mu\right)\right\|_{s}^{s} \leqq\|\mu\|_{1}^{s / s^{s}} \int_{T}\left\{\int_{T}|f(x+y)|^{s} F_{N}^{s}(x) d \lambda(x)\right\} d|\mu|(y)
$$

for any $s>1$, where $s^{\prime}=s(s-1)^{-1}$. Reference to (5.2.2) shows that this is $0(1)$, provided 


$$
\sup \sup _{N \in Z(\phi)} \int_{T}|f(x+y)|^{s} F_{N}^{s}(x) d \lambda(x)<\infty,
$$

which is verifiably the case whenever

$$
\sup _{y \in Z(\phi)}|f(y+x)|=O\left(|x|^{2}\right)
$$

with $\tau=s^{-2}(s-1)(2 s-1)$. But, since $f$ vanishes on $Z(\Phi)$, and since $5.1(1)$ ensures that $f \in \Lambda_{1 / p^{\prime}},(5.2 .12)$ will be satisfied as soon as we choose $s>1$ so close to 1 that $\tau \leqq 1 / p^{\prime}$.

Note added in proof. Concerning 4.3 , if $r \in(1,2), A^{r}$ is not an algebra under pointwise operations; see Lynette $M$. Butler, "Certain non-algebras in harmonic analysis" (to appear Bulletin Australian Math. Soc.).

\section{References}

[1] G. E. Shilov, 'On the Fourier coefficients of a class of continuous functions', (In Russian.) Doklady Akad. Nauk 35 (1942), 3-7.

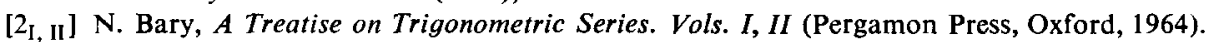
[3 I, II] R. E. Edwards, Fourier Series: A Modern Introduction (Vols. I, II. Holt, Rinehart \& Winston, Inc., New York, 1967, 1968).

[4] A. Zygmund, Trigonometric Series, Vol. I (Cambridge University Press, 1959).

[5] R. E. Edwards and E. Hewitt, 'Pointwise limits for sequences of convolution operators', Acta Math. 113 (1965), 181-218.

[6] R. E. Edwards, Functional Analysis: Theory and Applications (Holt, Rinehart and Winston, Inc., New York, 1965).

[7] Y. Katznelson, An Introduction to Harmonic Analysis (John Wiley and Sons, Inc., New York, 1968).

Department of Mathematics

Institute of Advanced Studies

ANU 